\title{
EXISTENCE RESULTS FOR A SYSTEM OF KIRCHHOFF-SCHRÖDINGER-MAXWELL EQUATIONS
}

\author{
LUCIO BOCCARDO, LUIGI ORSINA
}

To Vieri Benci, for his 70th birthday

\begin{abstract}
In this paper we study existence, nonexistence and properties of solutions for some Kirchhoff-Schrödinger-Maxwell systems as (1.3). The solutions can be seen as saddle points of functionals which are unbounded both from above and from below.
\end{abstract}

\section{INTRODUCTION}

Vieri Benci and Dino Fortunato (two great friends, and two important "starting blocks" of italian Nonlinear Analysis) studied in [3] a system of Schrödinger-Maxwell type, arising from the study of eigenvalue problems for the Schrödinger operator when coupled with the electromagnetic field. In that paper, dealing with the system

$$
\begin{cases}-\frac{1}{2} \Delta v+\varphi v=\omega v, & \text { in } \mathbb{R}^{N} \\ -\Delta \varphi=4 \pi v^{2}, & \text { in } \mathbb{R}^{N}\end{cases}
$$

they proved existence of an increasing and divergent sequence of eigenvalues $\left\{\omega_{n}\right\}$. Such a result was proved using variational methods, since solutions of (1.1) are critical points of a functional unbounded both from above and from below.

Systems like (1.1), but with a forcing term, were then studied in [6], [9] and [10]. In these papers, the authors dealt with problems of the form

$$
\left\{\begin{array}{l}
u \in W_{0}^{1,2}(\Omega):-\operatorname{div}(M(x) \nabla u)+A \psi|u|^{r-2} u=f(x), \\
\psi \in W_{0}^{1,2}(\Omega):-\operatorname{div}(M(x) \nabla \psi)=|u|^{r}
\end{array}\right.
$$

where $\Omega$ is a bounded, open subset of $\mathbb{R}^{N}$, with $N>2, M$ is a symmetric, bounded, and uniformly elliptic matrix, and $A>0$. The three papers studied existence and regularity of solutions for (1.2) under various assumptions on $r>1$, and $f$, a function belonging to $L^{m}(\Omega)$, with $m>1$. Among the results proved, of particular interest is the regularizing effect on the solutions, obtained thanks to the coupling of the equations in the system, with respect to the results separately known for the two equations of (1.2). For example, in [10] it is proved that if $f$ belongs to $L^{m}(\Omega)$, with $m \geq(r+1)^{\prime}=1+\frac{1}{r}$, then there exist solutions $u$ and $\psi$ both belonging to $W_{0}^{1,2}(\Omega)$. Since $r$ can be very large, this means for example that the first equation has a $W_{0}^{1,2}(\Omega)$ solution with a datum $f$ which is "almost" in $L^{1}(\Omega)$, and this is in sharp contrast with the known result for the first equation when considered by itself.

As in the paper by Benci and Fortunato, the solutions $u$ and $\psi$ given by [6], [9] and [10] can be seen, if $m \geq \frac{2 N}{N+2}$, as a critical point of saddle type for the indefinite 
functional

$$
J(v, \varphi)=\frac{1}{2} \int_{\Omega} M(x) \nabla v \nabla v-\frac{A}{2 r} \int_{\Omega} M(x) \nabla \varphi \nabla \varphi+\frac{A}{r} \int_{\Omega} \varphi^{+}|v|^{r}-\int_{\Omega} f v,
$$

defined for those functions $v$ and $\varphi$ in $W_{0}^{1,2}(\Omega)$ such that $\varphi^{+}|v|^{r}$ belongs to $L^{1}(\Omega)$ (and $+\infty$ otherwise).

In this paper, we deal with a system of Kirchhoff-Schrödinger-Maxwell type: more precisely, we will study both existence and properties of solutions for the system

$$
\left\{\begin{array}{l}
u \in W_{0}^{1,2}(\Omega):-\operatorname{div}\left(\left[a(x)+\int_{\Omega}|\nabla u|^{2}\right] \nabla u\right)+\psi|u|^{r-2} u=f(x), \\
\psi \in W_{0}^{1,2}(\Omega):-\operatorname{div}(M(x) \nabla \psi)=|u|^{r} .
\end{array}\right.
$$

We will suppose that $\Omega$ is a bounded open set of $\mathbb{R}^{N}, N>2$, that $r>1$, and that $f$ belongs to $L^{m}(\Omega)$, for some $m \geq 1$. Furthermore, the function $a: \Omega \rightarrow \mathbb{R}$ will be a measurable function such that there exist $0<\alpha \leq \beta$ such that

$$
0<\alpha \leq a(x) \leq \beta \quad \text { almost everywhere in } \Omega,
$$

while $M: \Omega \rightarrow \mathbb{R}^{N^{2}}$ will be a measurable matrix such that

$$
M(x) \xi \cdot \xi \geq \alpha|\xi|^{2}, \quad|M(x)| \leq \beta,
$$

for almost every $x$ in $\Omega$, and for every $\xi$ in $\mathbb{R}^{N}$.

Equations like

$$
-\operatorname{div}\left(\left[a(x)+\int_{\Omega}|\nabla u|^{2}\right] \nabla u\right)=f(x)
$$

have been introduced by G. Kirchhoff to describe the stationary states of nonhomegeneous elastic strings, and are therefore called elliptic Kirchhoff equations. Semilinear equations of Kirchhoff type have been studied for example in [1, 2, 12].

As for system (1.2), if $m \geq \frac{2 N}{N+2}$ the solutions of (1.3) can be seen as critical points of the (indefinite) functional

$$
J(v, \varphi)=\left\{\begin{array}{cc}
\frac{1}{2} \int_{\Omega} a(x)|\nabla v|^{2}+\frac{1}{4}\left[\int_{\Omega}|\nabla v|^{2}\right]^{2}-\frac{1}{2 r} \int_{\Omega} M(x) \nabla \varphi \nabla \varphi & \text { if } \varphi^{+}|v|^{r} \in L^{1}(\Omega), \\
+\frac{1}{r} \int_{\Omega} \varphi^{+}|v|^{r}-\int_{\Omega} f v, & \text { otherwise. }
\end{array}\right.
$$

The main difference between systems (1.2) and (1.3) is the presence of the nonlocal Kirchhoff-type term

$$
-\operatorname{div}\left(\left[\int_{\Omega}|\nabla u|^{2}\right] \nabla u\right)
$$

As we will prove, this term will be "responsible" for an alternative-like existence result: either the assumptions on $r$ and $m$ are such that there exists solutions $u$ and $\psi$ in $W_{0}^{1,2}(\Omega)$ of the system, or, when trying to prove existence of solutions by approximation, the system degenerates to a single, different equation (see. Section 3).

[such solutions do not exist, and the system degenerates, as the result of an approximation technique, to a single, different equation.]

The plan of the paper is as follows: in Section 2 we will prove existence of solutions for system (1.3) under various assumptions on $r$ and $m$; in order to prove existence, we will consider a sequence of approximating systems, for which existence of solutions will 
be proved in the Appendix: various a priori estimates on the sequence of solutions of these systems, and some strong compactness results will then allow us to pass to the limit and prove existence of solutions for system (1.3). We will also prove that, under some assumptions on $r$ and $m$, the solutions of system (1.3) can be seen as saddle points of the functional $J$ defined in (1.6).

In Section 3 we will prove some nonexistence results if the assumptions on the data of the problem are not enough in order to prove finite energy solutions (i.e., in $W_{0}^{1,2}(\Omega)$ ). More precisely, we will prove that if $\left\{u_{n}\right\}$ and $\left\{\psi_{n}\right\}$ are sequences of solutions of some systems which approximate (1.3), then the norm of $u_{n}$ in $W_{0}^{1,2}(\Omega)$ diverges, the sequence $\left\{u_{n}\right\}$ converges to zero in a larger space than $W_{0}^{1,2}(\Omega)$, and the sequence $\left\{v_{n}\right\}$ defined as

$$
v_{n}=\left[\int_{\Omega}\left|\nabla u_{n}\right|^{2}\right] u_{n},
$$

converges to the entropy solution $w$ (see [4]) of the equation $-\Delta w=f$. As for the sequence $\left\{\psi_{n}\right\}$ it also tends to zero.

In the whole paper, we will frequently make use of the following two functions of one real variable $s$, depending on a parameter $k>0$ :

$$
T_{k}(s)=\max (-k, \min (s, k)), \quad G_{k}(s)=s-T_{k}(s)=(|s|-k)^{+} \operatorname{sgn}(s) .
$$

\section{Existence in the General CASE}

As stated in the introduction, we will prove existence of solutions for the (KirchhoffSchrödinger-Maxwell) system

$$
\left\{\begin{array}{l}
u \in W_{0}^{1,2}(\Omega):-\operatorname{div}\left(\left[a(x)+\int_{\Omega}|\nabla u|^{2}\right] \nabla u\right)+\psi|u|^{r-2} u=f, \\
\psi \in W_{0}^{1,2}(\Omega):-\operatorname{div}(M(x) \nabla \psi)=|u|^{r},
\end{array}\right.
$$

under different assumptions on $r$ and $m$. Our result is the following.

Theorem 2.1. Let $a$ and $M$ be such that (1.4) and (1.5) hold. Let $r>1$ and let $f$ in $L^{m}(\Omega)$. We have the following:

(i) if $r \geq \frac{N+2}{N-2}$, and if $m \geq(r+1)^{\prime}$, there exist $u$ and $\psi$ in $W_{0}^{1,2}(\Omega)$, solutions of (2.1); furthermore,

(a) if $m>\frac{N}{2}$ then $u$ belongs to $L^{\infty}(\Omega)$;

(b) if $m=\frac{N}{2}$ then $u$ has exponential summability and belongs to $L^{p}(\Omega)$ for every $p>1$;

(c) if $(r+1)^{\prime} \leq m<\frac{N}{2}$, then $u$ belongs to $L^{\sigma}(\Omega)$, with $\sigma=\max \left(m^{* *}, \frac{m(2 r+1)}{m+1}\right)$, where $m^{* *}=\frac{N m}{N-2 m}$;

(ii) if $1<r<\frac{N+2}{N-2}$, and if $m \geq \frac{2 N}{N+2}$, there exist $u$ and $\psi$ in $W_{0}^{1,2}(\Omega)$, solutions of (2.1); furthermore,

(a) if $m>\frac{N}{2}$ then $u$ belongs to $L^{\infty}(\Omega)$;

(b) if $m=\frac{N}{2}$ then $u$ has exponential summability and belongs to $L^{p}(\Omega)$ for every $p>1$;

(c) if $\frac{2 N}{N+2} \leq m<\frac{N}{2}$, then $u$ belongs to $L^{m^{* *}}(\Omega)$, where $m^{* *}=\frac{N m}{N-2 m}$. 


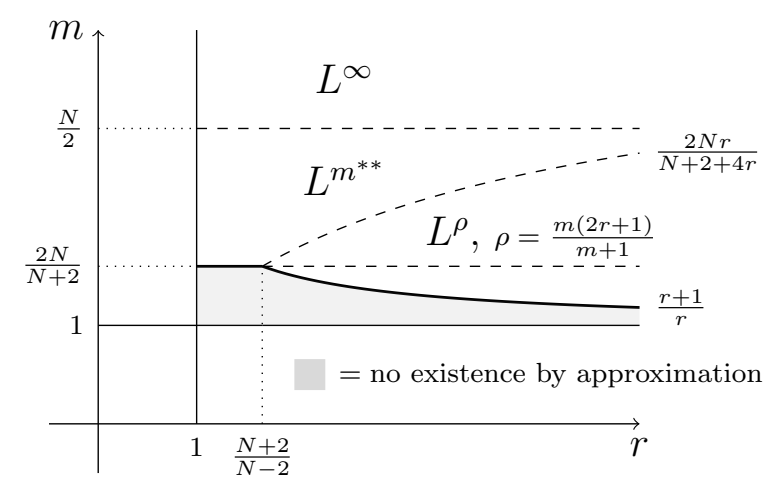

Figure 1: Regularity for $u$ in Lebesgue spaces as a function of $r$ and $m$ as a consequence of Theorem 2.1.

In Figure 1, the dashed line $m=\frac{2 N r}{N+2+4 r}$ divides the two zones where $m^{* *}>\frac{m(2 r+1)}{m+1}$, or $m^{* *}<\frac{m(2 r+1)}{m+1}$. The white zone below the dashed line $m=\frac{2 N}{N+2}$, and above the curve $m=\frac{r+1}{r}$ is where the regularizing effect with respect to the single equation happens: even though the datum is not in the dual space of $W_{0}^{1,2}(\Omega)$, the solutions belong to $W_{0}^{1,2}(\Omega)$.

The proof will be divided in several steps, dealing with the various possible values for $r$ and $m$. We begin with existence of approximating solutions.

Approximate solutions. Let $n$ in $\mathbb{N}$, and let $f_{n}=T_{n}(f)$, so that $\left\{f_{n}\right\}$ is a sequence of $L^{\infty}(\Omega)$ functions which strongly converges to $f$ in $L^{m}(\Omega)$, and satisfies the inequality $\left|f_{n}\right| \leq|f|$. Thanks to Theorem 3.4 (see the Appendix), for every $n$ in $\mathbb{N}$ there exist weak solutions $u_{n}$ and $\psi_{n}$ in $W_{0}^{1,2}(\Omega) \cap L^{\infty}(\Omega)$, with $\psi_{n} \geq 0$, of the approximate system

$$
\left\{\begin{array}{l}
u_{n} \in W_{0}^{1,2}(\Omega):-\operatorname{div}\left(\left[a(x)+\int_{\Omega}\left|\nabla u_{n}\right|^{2}\right] \nabla u_{n}\right)+\psi_{n}\left|u_{n}\right|^{r-2} u_{n}=f_{n}, \\
\psi_{n} \in W_{0}^{1,2}(\Omega):-\operatorname{div}\left(M(x) \nabla \psi_{n}\right)=\left|u_{n}\right|^{r}
\end{array}\right.
$$

$L^{\infty}(\Omega)$ And EXPonential EStimates. Suppose that $m \geq \frac{N}{2}$, and choose $G_{k}\left(u_{n}\right)$ as test function in the first equation of (2.2). Dropping two positive terms, and using (1.4), we obtain

$$
\alpha \int_{\Omega}\left|\nabla G_{k}\left(u_{n}\right)\right|^{2} \leq \int_{\Omega}\left|f_{n}\right|\left|G_{k}\left(u_{n}\right)\right|
$$

which is the starting point of either the $L^{\infty}(\Omega)$ estimate by Stampacchia (see [11], Théoreème 4.1) if $m>\frac{N}{2}$, or the exponential estimate if $m=\frac{N}{2}$. That is, under the assumption that $m>\frac{N}{2}$, there exists a constant $C>0$ such that

$$
\left\|u_{n}\right\|_{L^{\infty}(\Omega)} \leq C\|f\|_{L^{m}(\Omega)},
$$

while if $m=\frac{N}{2}$ the sequence $\left\{u_{n}\right\}$ has exponential summability (uniformly with respect to $n$ ), so that it is bounded in $L^{p}(\Omega)$, for every $p>1$. From this estimates, it is easy to prove that the sequence $\left\{u_{n}\right\}$ is bounded in $W_{0}^{1,2}(\Omega)$ (choose $u_{n}$ as test function, use (1.4) and drop two positive terms), and that the sequence $\left\{\psi_{n}\right\}$ is bounded in 
$W_{0}^{1,2}(\Omega) \cap L^{\infty}(\Omega)$ (use (1.5) and Stampacchia's result as above since the sequence $\left\{\left|u_{n}\right|^{r}\right\}$ is bounded in $L^{s}(\Omega)$, for some $\left.s>\frac{N}{2}\right)$.

ESTIMATES WHICH USE THE LOWER ORDER TERM. In this step, we will suppose that $m \geq(r+1)^{\prime}$. Choosing $u_{n}$ as test function in the first equation of (2.2), using (1.4) and dropping a positive term, we obtain

$$
\alpha \int_{\Omega}\left|\nabla u_{n}\right|^{2}+\int_{\Omega} \psi_{n}\left|u_{n}\right|^{r} \leq \int_{\Omega} f_{n} u_{n}
$$

while choosing $\psi_{n}$ as test function in the second equation we obtain, using (1.5),

$$
\alpha \int_{\Omega}\left|\nabla \psi_{n}\right|^{2} \leq \int_{\Omega} \psi_{n}\left|u_{n}\right|^{r}
$$

Therefore, we have that

$$
\alpha \int_{\Omega}\left|\nabla u_{n}\right|^{2}+\alpha \int_{\Omega}\left|\nabla \psi_{n}\right|^{2} \leq \int_{\Omega}\left|f_{n}\right|\left|u_{n}\right|
$$

We now follow [10]: let $\gamma \geq 1$ to be determined later, and choose $\left|u_{n}\right|^{2 \gamma-2} u_{n}$ as test function in the first equation of (2.2); using (1.4), and dropping two positive terms, we obtain, since $\left|f_{n}\right| \leq|f|$,

$$
\alpha(2 \gamma-1) \int_{\Omega}\left|\nabla u_{n}\right|^{2}\left|u_{n}\right|^{2 \gamma-2} \leq \int_{\Omega}\left|f_{n}\right|\left|u_{n}\right|^{2 \gamma-1} \leq \int_{\Omega}|f|\left|u_{n}\right|^{2 \gamma-1} .
$$

On the other hand, choosing $\left|u_{n}\right|^{\gamma}$ as test function in the second equation we obtain, using Young inequality and (1.5),

$$
\begin{aligned}
\int_{\Omega}\left|u_{n}\right|^{r+\gamma} & =\gamma \int_{\Omega} M(x) \nabla \psi_{n} \nabla u_{n}\left|u_{n}\right|^{\gamma-1} \operatorname{sgn}\left(u_{n}\right) \\
& \leq \beta \gamma \int_{\Omega}\left|\nabla \psi_{n}\right|\left|\nabla u_{n}\right|\left|u_{n}\right|^{\gamma-1} \leq C \int_{\Omega}\left|\nabla \psi_{n}\right|^{2}+C \int_{\Omega}\left|\nabla u_{n}\right|^{2}\left|u_{n}\right|^{2 \gamma-2} .
\end{aligned}
$$

Using (2.3) and (2.4) with this inequality, we obtain

$$
\int_{\Omega}\left|u_{n}\right|^{r+\gamma} \leq C \int_{\Omega}|f|\left|u_{n}\right|+C \int_{\Omega}|f|\left|u_{n}\right|^{2 \gamma-1}
$$

which implies that

$$
\int_{\Omega}\left|u_{n}\right|^{r+\gamma} \leq C \int_{\Omega}|f|\left|u_{n}\right|+C \int_{\Omega}|f|\left|u_{n}\right|^{2 \gamma-1} \leq C \int_{\Omega}|f|+C \int_{\Omega}|f|\left|u_{n}\right|^{2 \gamma-1},
$$

where in the last passage we have used that $2 \gamma-1 \geq 1$ since $\gamma \geq 1$. We now choose $\gamma=\frac{r(m-1)+m}{m+1}$, so that $\gamma \geq 1$ since $m \geq \frac{r+1}{r}=(r+1)^{\prime}$. With this choice of $\gamma$, we have $r+\gamma=\frac{m(2 r+1)}{m+1}=(2 \gamma-1) m^{\prime}$, so that from (2.5) we obtain, using Hölder inequality,

$$
\int_{\Omega}\left|u_{n}\right|^{\frac{m(2 r+1)}{m+1}} \leq C\|f\|_{L^{1}(\Omega)}+C\|f\|_{m}\left[\int_{\Omega}\left|u_{n}\right|^{\frac{m(2 r+1)}{m+1}}\right]^{\frac{1}{m^{\prime}}} .
$$

Recalling that $m>1$, we therefore obtain (after simplfying equal terms) that

$$
\left[\int_{\Omega}\left|u_{n}\right|^{\frac{m(2 r+1)}{m+1}}\right]^{\frac{1}{m}} \leq C\|f\|_{L^{m}(\Omega)},
$$


that is, the sequence $\left\{u_{n}\right\}$ is bounded in $L^{s}(\Omega)$, with $s=\frac{m(2 r+1)}{m+1}$. As a consequence of this estimate, and of the fact that $s \geq m^{\prime}$, we have that

$$
\int_{\Omega}|f|\left|u_{n}\right| \leq C
$$

so that from (2.3) it follows that the sequences $\left\{u_{n}\right\}$ and $\left\{\psi_{n}\right\}$ are bounded in $W_{0}^{1,2}(\Omega)$.

ESTIMATES NOT USING THE LOWER ORDER TERM. In this step, we will suppose that $m \geq \frac{2 N}{N+2}$. Let $u_{n}$ and $\psi_{n}$ be solutions of (2.2), let $\gamma \geq 1$, and choose $\left|u_{n}\right|^{2 \gamma-2} u_{n}$ as test function in the first equation, to obtain, after using (1.4), and dropping two positive terms,

$$
\alpha(2 \gamma-1) \int_{\Omega}\left|\nabla u_{n}\right|^{2}\left|u_{n}\right|^{2 \gamma-2} \leq \int_{\Omega} f_{n}\left|u_{n}\right|^{2 \gamma-2} u_{n}
$$

Thanks to Sobolev and Hölder inequalities, and to the assumption $\left|f_{n}\right| \leq|f|$, we have

$$
\begin{aligned}
\frac{\alpha \mathcal{S}(2 \gamma-1)}{\gamma^{2}}\left[\int_{\Omega}\left|u_{n}\right|^{2^{*} \gamma}\right]^{\frac{2}{2^{*}}} & \leq \alpha(2 \gamma-1) \int_{\Omega}\left|\nabla u_{n}\right|^{2} u_{n}^{2 \gamma-2} \\
& \leq \int_{\Omega} f_{n}\left|u_{n}\right|^{2 \gamma-2} u_{n} \leq\|f\|_{L^{m}(\Omega)}\left[\int_{\Omega}\left|u_{n}\right|^{(2 \gamma-1) m^{\prime}}\right]^{\frac{1}{m^{\prime}}} .
\end{aligned}
$$

Choose now $\gamma=\frac{m^{* *}}{2^{*}}$, so that $\gamma \geq 1\left(\right.$ since $\left.m^{* *} \geq(2 N / N+2)^{* *}=2^{*}\right)$ and $(2 \gamma-1) m^{\prime}=$ $m^{* *}$; we obtain

$$
\left[\int_{\Omega}\left|u_{n}\right|^{m^{* *}}\right]^{\frac{2}{2^{*}}} \leq C\|f\|_{L^{m}(\Omega)}\left[\int_{\Omega}\left|u_{n}\right|^{m^{* *}}\right]^{\frac{1}{m^{\prime}}}
$$

which can be rewritten, after simplifying equal terms, as

$$
\left[\int_{\Omega}\left|u_{n}\right|^{m^{* *}}\right]^{\frac{1}{m^{* *}}} \leq C\|f\|_{L^{m}(\Omega)} .
$$

Thus, the sequence $\left\{u_{n}\right\}$ is bounded in $L^{m^{* *}}(\Omega)$; since

$$
\frac{1}{m}+\frac{1}{m^{* *}}=\frac{1}{m}+\frac{1}{m}-\frac{2}{N}=\frac{2}{m^{*}} \leq 1
$$

being $m \geq \frac{2 N}{N+2}$, we have that the sequence $\left\{f_{n} u_{n}\right\}$ is bounded in $L^{1}(\Omega)$. Therefore, choosing $u_{n}$ as test function in the first equation of (2.2), we have (dropping a positive term, and using (1.4)) that

$$
\alpha \int_{\Omega}\left|\nabla u_{n}\right|^{2}+\int_{\Omega} \psi_{n}\left|u_{n}\right|^{r} \leq \int_{\Omega} f_{n} u_{n} \leq C
$$

that is, the sequence $\left\{u_{n}\right\}$ is bounded in $W_{0}^{1,2}(\Omega)$, and the sequence $\left\{\psi_{n}\left|u_{n}\right|^{r}\right\}$ is bounded in $L^{1}(\Omega)$. Choosing $\psi_{n}$ as test function in the second equation of (2.2), and using (1.5), we thus have

$$
\alpha \int_{\Omega}\left|\nabla \psi_{n}\right|^{2} \leq \int_{\Omega} \psi_{n}\left|u_{n}\right|^{r} \leq C,
$$

so that also the sequence $\left\{\psi_{n}\right\}$ is bounded in $W_{0}^{1,2}(\Omega)$.

END OF THE PROOF. As a consequence of the previous proofs, and under the assumptions that $m \geq \max \left((r+1)^{\prime}, \frac{2 N}{N+2}\right)$ and $r>1$, we have that:

- the sequences $\left\{u_{n}\right\}$ and $\left\{\psi_{n}\right\}$ are bounded in $W_{0}^{1,2}(\Omega)$; 
- the sequence $\left\{u_{n}\right\}$ is bounded in $L^{\sigma}(\Omega)$, with $\sigma=\max \left(m^{* *}, \frac{m(2 r+1)}{m+1}\right)$ if $m<\frac{N}{2}$, and $\sigma=+\infty$ if $m>\frac{N}{2}$.

Therefore, up to subsequences, $u_{n}$ converges, weakly in $W_{0}^{1,2}(\Omega)$, weakly in $L^{\sigma}(\Omega)$, and almost everywhere in $\Omega$, to some function $u$, while $\psi_{n}$ converges, waekly in $W_{0}^{1,2}(\Omega)$ and almost everywhere in $\Omega$, to some function $\psi$. Since the sequence $\left\{\left|u_{n}\right|^{r}\right\}$ is bounded in $L^{\rho}(\Omega)$, with $\rho=\frac{\sigma}{r}>1$, it is weakly convergent in the same space to $|u|^{r}$. Therefore, one can pass to the limit in the identities

$$
\int_{\Omega} M(x) \nabla \psi_{n} \nabla w=\int_{\Omega}\left|u_{n}\right|^{r} w, \quad \forall w \in W_{0}^{1,2}(\Omega) \cap L^{\infty}(\Omega),
$$

to have that $\psi$ and $u$ are such that

$$
\int_{\Omega} M(x) \nabla \psi \nabla w=\int_{\Omega}|u|^{r} w, \quad \forall w \in W_{0}^{1,2}(\Omega) \cap L^{\infty}(\Omega) .
$$

Choosing $w=T_{k}(v)$, with $v \geq 0$ in $W_{0}^{1,2}(\Omega)$, we have that

$$
\int_{\Omega} M(x) \nabla \psi \nabla T_{k}(v)=\int_{\Omega}|u|^{r} T_{k}(v), \quad \forall k>0 .
$$

Passing to the limit as $k$ tends to infinity, using Lebesgue theorem in the left hand side (recall that $\psi$ belongs to $W_{0}^{1,2}(\Omega)$ ), and Beppo Levi theorem in the right hand side, we have that

$$
\int_{\Omega} M(x) \nabla \psi \nabla v=\int_{\Omega}|u|^{r} v, \quad \forall v \in W_{0}^{1,2}(\Omega), v \geq 0 .
$$

If $v$ belongs to $W_{0}^{1,2}(\Omega)$, writing $v=v^{+}-v^{-}$, and subtracting the above identities written for $v^{+}$and $v^{-}$(not that both terms are finite, because the left hand side is finite), we have that

$$
\int_{\Omega} M(x) \nabla \psi \nabla v=\int_{\Omega}|u|^{r} v, \quad \forall v \in W_{0}^{1,2}(\Omega)
$$

that is, $\psi$ is a weak solution of the second equation.

We study now the first equation: due to the presence of the term $\int_{\Omega}\left|\nabla u_{n}\right|^{2}$, weak convergence in $W_{0}^{1,2}(\Omega)$ is not enough in order to pass to the limit, as it is not enough the boundedness of $\psi_{n}\left|u_{n}\right|^{r}$ in $L^{1}(\Omega)$. Therefore, we need some extra work. First of all, let $\varepsilon>0, k>0$, and choose $\frac{1}{\varepsilon} u_{n}^{+} T_{\varepsilon}\left(G_{k}\left(u_{n}\right)\right)$ as test function in the first equation of the system. Dropping two positive terms (those coming from the differential part of the equation), and using that $\left|f_{n}\right| \leq|f|$, we obtain

$$
\frac{1}{\varepsilon} \int_{\left\{u_{n} \geq k\right\}} \psi_{n}\left[u_{n}^{+}\right]^{r} T_{\varepsilon}\left(G_{k}\left(u_{n}\right)\right) \leq \frac{1}{\varepsilon} \int_{\left\{u_{n} \geq k\right\}}\left|f_{n}\right|\left|u_{n}\right|\left|T_{\varepsilon}\left(G_{k}\left(u_{n}\right)\right)\right| \leq \frac{1}{\varepsilon} \int_{\left\{u_{n} \geq k\right\}}|f|\left|u_{n}\right|\left|T_{\varepsilon}\left(G_{k}\left(u_{n}\right)\right)\right| .
$$

Letting $\varepsilon$ tend to zero, using Fatou lemma on the left hand side, and Lebesgue theorem on the right hand one (recall that every $u_{n}$ is a function in $L^{\infty}(\Omega)$ ), we have that

$$
\int_{\left\{u_{n} \geq k\right\}} \psi_{n}\left[u_{n}^{+}\right]^{r} \leq \int_{\left\{u_{n} \geq k\right\}}|f|\left|u_{n}\right| \leq\left[\int_{\left\{u_{n} \geq k\right\}}|f|^{m}\right]^{\frac{1}{m}}\left\|u_{n}\right\|_{L^{m^{\prime}}(\Omega)} \leq C\left[\int_{\left\{u_{n} \geq k\right\}}|f|^{m}\right]^{\frac{1}{m}},
$$

since the sequence $\left\{u_{n}\right\}$ is bounded in $L^{m^{\prime}}(\Omega)$ being $\sigma \geq m^{\prime}$. Analogous calculations imply that

$$
\int_{\left\{u_{n} \leq-k\right\}} \psi_{n}\left[u_{n}^{-}\right]^{r} \leq C\left[\int_{\left\{u_{n} \leq-k\right\}}|f|^{m}\right]^{\frac{1}{m}},
$$


so that

$$
\int_{\left\{\left|u_{n}\right| \geq k\right\}} \psi_{n}\left|u_{n}\right|^{r} \leq C\left[\int_{\left\{\left|u_{n}\right| \geq k\right\}}|f|^{m}\right]^{\frac{1}{m}}
$$

Let now $E$ be a measurable subset of $\Omega$. Then

$$
\begin{aligned}
\int_{E} \psi_{n}\left|u_{n}\right|^{r} & =\int_{E \cap\left\{\left|u_{n}\right| \leq k\right\}} \psi_{n}\left|u_{n}\right|^{r}+\int_{E \cap\left\{\left|u_{n}\right| \geq k\right\}} \psi_{n}\left|u_{n}\right|^{r} \\
& \leq k^{r} \int_{E} \psi_{n}+C\left[\int_{\left\{\left|u_{n}\right| \geq k\right\}}|f|^{m}\right]^{\frac{1}{m}} .
\end{aligned}
$$

Let now $\varepsilon>0$, and choose $k$ large enough so that

$$
C\left[\int_{\left\{\left|u_{n}\right| \geq k\right\}}|f|^{m}\right]^{\frac{1}{m}} \leq \varepsilon, \quad \forall n \in \mathbb{N} .
$$

Such a choice of $k$ is possible since the measure of $\left\{\left|u_{n}\right| \geq k\right\}$ tends to zero as $k$ tends to infinity, uniformly in $n$, as a consequence of the boundedness of $\left\{u_{n}\right\}$ in (for example) $L^{1}(\Omega)$, and since $|f|^{m}$ belongs to $L^{1}(\Omega)$. Once $k$ has been chosen, let $\delta>0$ be such that meas $(E) \leq \delta$ implies that

$$
k^{r} \int_{E} \psi_{n} \leq \varepsilon, \quad \forall n \in \mathbb{N} .
$$

Such a choice of $\delta$ is possible thanks to Vitali theorem since the sequence $\left\{\psi_{n}\right\}$ is strongly convergent in (at least) $L^{1}(\Omega)$ being bounded in $W_{0}^{1,2}(\Omega)$. Thus, the sequence $\left\{\psi_{n}\left|u_{n}\right|^{r}\right\}$ is uniformly equiintegrable. Since it is almost everywhere convergent, Vitali theorem implies that

$$
\psi_{n}\left|u_{n}\right|^{r} \text { strongly converges to } \psi|u|^{r} \text { in } L^{1}(\Omega) \text {. }
$$

With the same technique, one can prove that the sequence $\left\{\psi_{n}\left|u_{n}\right|^{r-1}\right\}$ is uniformly equiintegrable, so that

$$
\psi_{n}\left|u_{n}\right|^{r-1} \text { strongly converges to } \psi|u|^{r-1} \text { in } L^{1}(\Omega) .
$$

Define now

$$
D_{n}=\int_{\Omega}\left|\nabla u_{n}\right|^{2}
$$

Since the sequence $\left\{u_{n}\right\}$ is bounded in $W_{0}^{1,2}(\Omega),\left\{D_{n}\right\}$ is a bounded sequence of real numbers, which we may suppose converges to some real number $D$. Let $k>0$ (to be determined later), and choose $u_{n}-T_{k}(u)$ as test function in the first equation of the system, to obtain

$$
\int_{\Omega}\left[a(x)+D_{n}\right] \nabla u_{n} \nabla\left(u_{n}-T_{k}(u)\right)+\int_{\Omega} \psi_{n}\left|u_{n}\right|^{r-2} u_{n}\left(u_{n}-T_{k}(u)\right)=\int_{\Omega} f_{n}\left(u_{n}-T_{k}(u)\right) .
$$

Adding and subtracting the term

$$
\int_{\Omega}\left[a(x)+D_{n}\right] \nabla T_{k}(u) \nabla\left(u_{n}-T_{k}(u)\right),
$$

we obtain, using (1.4) and dropping a positive term,

$$
\begin{aligned}
\alpha \int_{\Omega} & \left|\nabla\left(u_{n}-T_{k}(u)\right)\right|^{2}+\int_{\Omega} \psi_{n}\left|u_{n}\right|^{r-2} u_{n}\left(u_{n}-T_{k}(u)\right) \\
\leq & \int_{\Omega} f\left(u_{n}-T_{k}(u)\right)+\int_{\Omega}\left[a(x)+D_{n}\right] \nabla T_{k}(u) \nabla\left(u_{n}-T_{k}(u)\right) .
\end{aligned}
$$


Since $f_{n}$ is strongly convergent to $f$ in $L^{m}(\Omega)$, and $u_{n}-T_{k}(u)$ is weakly convergent in (at least) $L^{m^{\prime}}(\Omega)$ (recall that $\sigma \geq m^{\prime}$ ), we have that

$$
\lim _{n \rightarrow+\infty} \int_{\Omega} f_{n}\left(u_{n}-T_{k}(u)\right)=\int_{\Omega} f G_{k}(u) .
$$

Thanks to both (2.7) and (2.8), we also have that

$$
\lim _{n \rightarrow+\infty} \int_{\Omega} \psi_{n}\left|u_{n}\right|^{r-2} u_{n}\left(u_{n}-T_{k}(u)\right)=\int_{\Omega} \psi|u|^{r-2} u G_{k}(u) \geq 0,
$$

while the boundedness of $\left\{u_{n}\right\}$ in $W_{0}^{1,2}(\Omega)$, the weak convergence of $\nabla\left(u_{n}-T_{k}(u)\right)$ to $\nabla G_{k}(u)$ in $\left(L^{2}(\Omega)\right)^{N}$ and (1.4) imply that

$$
\lim _{n \rightarrow+\infty} \int_{\Omega}\left[a(x)+D_{n}\right] \nabla T_{k}(u) \nabla\left(u_{n}-T_{k}(u)\right)=\int_{\Omega}[a(x)+D] \nabla T_{k}(u) \nabla G_{k}(u)=0,
$$

since $\nabla T_{k}(u) \nabla G_{k}(u) \equiv 0$ almost everywhere in $\Omega$. Therefore, we have that

$$
\limsup _{n \rightarrow+\infty} \alpha \int_{\Omega}\left|\nabla\left(u_{n}-T_{k}(u)\right)\right|^{2} \leq \int_{\Omega} f G_{k}(u) .
$$

Since $u=T_{k}(u)+G_{k}(u)$, we have

$$
\int_{\Omega}\left|\nabla\left(u_{n}-u\right)\right|^{2} \leq 2 \int_{\Omega}\left|\nabla\left(u_{n}-T_{k}(u)\right)\right|^{2}+2 \int_{\Omega}\left|\nabla G_{k}(u)\right|^{2} .
$$

Therefore, using (2.9), we obtain

$$
\begin{aligned}
\limsup _{n \rightarrow+\infty} \int_{\Omega}\left|\nabla\left(u_{n}-u\right)\right|^{2} & \leq 2 \limsup _{n \rightarrow+\infty} \int_{\Omega}\left|\nabla\left(u_{n}-T_{k}(u)\right)\right|^{2}+2 \int_{\Omega}\left|\nabla G_{k}(u)\right|^{2} \\
& \leq \frac{2}{\alpha} \int_{\Omega} f G_{k}(u)+2 \int_{\Omega}\left|\nabla G_{k}(u)\right|^{2} .
\end{aligned}
$$

Since $f u$ belongs to $L^{1}(\Omega)$, and $|\nabla u|$ belongs to $L^{2}(\Omega)$, given $\varepsilon>0$ there exists $k=$ $k_{\varepsilon}>0$ such that

$$
\frac{2}{\alpha} \int_{\Omega} f G_{k}(u)+2 \int_{\Omega}\left|\nabla G_{k}(u)\right|^{2} \leq \varepsilon,
$$

so that

$$
0 \leq \limsup _{n \rightarrow+\infty} \int_{\Omega}\left|\nabla\left(u_{n}-u\right)\right|^{2} \leq \varepsilon
$$

Being $\varepsilon>0$ arbitrary, this implies that $u_{n}$ is strongly convergent to $u$ in $W_{0}^{1,2}(\Omega)$. Hence,

$$
D=\lim _{n \rightarrow+\infty} D_{n}=\lim _{n \rightarrow+\infty} \int_{\Omega}\left|\nabla u_{n}\right|^{2}=\int_{\Omega}|\nabla u|^{2},
$$

and (using also (2.8)) we can pass to the limit in the identities

$\int_{\Omega}\left[a(x)+\int_{\Omega}\left|\nabla u_{n}\right|^{2}\right] \nabla u_{n} \nabla \eta+\int_{\Omega} \psi_{n}\left|u_{n}\right|^{r-2} u_{n} \eta=\int_{\Omega} f_{n} \eta, \quad \forall \eta \in W_{0}^{1,2}(\Omega) \cap L^{\infty}(\Omega)$,

to have that

$$
\int_{\Omega}\left[a(x)+\int_{\Omega}|\nabla u|^{2}\right] \nabla u \nabla \eta+\int_{\Omega} \psi|u|^{r-2} u \eta=\int_{\Omega} f \eta, \quad \forall \eta \in W_{0}^{1,2}(\Omega) \cap L^{\infty}(\Omega),
$$

as desired. 
REMARK 2.2. If $1<r \leq \frac{N+2}{N-2}$, and $f$ belongs to $L^{m}(\Omega)$, with $m \geq \frac{2 N}{N+2}$, then not only $\psi$ but also $u$ is a weak solution of the first equation of (2.1). Indeed, since both $u$ and $\psi$ belong to $L^{2^{*}}(\Omega)$ (being $W_{0}^{1,2}(\Omega)$ functions), we have that

$$
\psi|u|^{r-2} u \in L^{\rho}(\Omega), \quad \rho=\frac{2^{*}}{r} .
$$

Since, by the assumptions on $r$,

$$
\frac{2^{*}}{r} \geq \frac{2 N}{N-2} \frac{N-2}{N+2}=\frac{2 N}{N+2}
$$

the function $\psi|u|^{r-2} u$ belongs to the dual of $W_{0}^{1,2}(\Omega)$; therefore, one has (by density of $W_{0}^{1,2}(\Omega) \cap L^{\infty}(\Omega)$ in $\left.W_{0}^{1,2}(\Omega)\right)$

$$
\int_{\Omega}\left[a(x)+\int_{\Omega}|\nabla u|^{2}\right] \nabla u \nabla \varphi+\int_{\Omega} \psi|u|^{r-2} u \varphi=\int_{\Omega} f \varphi, \quad \forall \varphi \in W_{0}^{1,2}(\Omega) .
$$

as desired.

Thanks to this remark, we can prove that the solution $(u, \psi)$ of system $(2.1)$ given by Theorem 2.1 can be seen (under some assumptions on $r$ and $f$ ) as a saddle point of a suitable functional.

Theorem 2.3. Suppose that $a$ and $M$ satisfy (1.4) and (1.5), and that $M$ is symmetric. Let $1<r \leq \frac{2 N}{N+2}$ and let $f$ in $L^{m}(\Omega)$, with $m \geq \frac{2 N}{N+2}$. Then the solution $(u, \psi)$ of system (2.1) given by Theorem 2.1 is a saddle point of the functional $J$ defined in (1.6), that is

$$
J(u, \varphi) \leq J(u, \psi) \leq J(v, \psi), \quad \forall v, \varphi \in W_{0}^{1,2}(\Omega) \text { such that } \psi|v|^{r} \in L^{1}(\Omega) .
$$

Proof. We begin with the second equation of (2.1); by Theorem $2.1, \psi$ is a weak solution of the second equation of (2.1). Choosing $\frac{\psi-\varphi^{+}}{r}$, with $\varphi$ in $W_{0}^{1,2}(\Omega)$, as test function, we obtain

$$
\frac{1}{r} \int_{\Omega} M(x) \nabla \psi \nabla\left(\psi-\varphi^{+}\right)=\frac{1}{r} \int_{\Omega}|u|^{r}\left(\psi-\varphi^{+}\right) .
$$

Adding and subtracting the term

$$
\frac{1}{2 r} \int_{\Omega} M(x) \nabla \varphi^{+} \nabla \varphi^{+}
$$

we obtain, after straightforward passages,

$$
\begin{aligned}
& \frac{1}{2 r} \int_{\Omega} M(x) \nabla\left(\psi-\varphi^{+}\right) \nabla\left(\psi-\varphi^{+}\right)+\frac{1}{2 r} \int_{\Omega} M(x) \nabla \psi \nabla \psi-\frac{1}{r} \int_{\Omega} \psi|u|^{r} \\
& \quad=\frac{1}{2 r} \int_{\Omega} M(x) \nabla \varphi^{+} \nabla \varphi^{+}-\frac{1}{r} \int_{\Omega} \varphi^{+}|u|^{r} .
\end{aligned}
$$

Since the first term is positive, we therefore have that (recall that $\psi \geq 0$, so that $\left.\psi=\psi^{+}\right)$

$$
\frac{1}{2 r} \int_{\Omega} M(x) \nabla \psi \nabla \psi-\frac{1}{r} \int_{\Omega} \psi^{+}|u|^{r} \leq \frac{1}{2 r} \int_{\Omega} M(x) \nabla \varphi^{+} \nabla \varphi^{+}-\frac{1}{r} \int_{\Omega} \varphi^{+}|u|^{r},
$$

for every $\varphi$ in $W_{0}^{1,2}(\Omega)$. Changing sign to this identity, and adding to both sides the (finite, thanks to the assumptions on $f$ and to the fact that $u$ belongs to $W_{0}^{1,2}(\Omega)$ ) term

$$
\frac{1}{2} \int_{\Omega} a(x)|\nabla u|^{2}+\frac{1}{2}\left[\int_{\Omega}|\nabla u|^{2}\right]^{2}-\int_{\Omega} f u
$$


we have that

$$
J(u, \varphi) \leq J(u, \psi), \quad \forall \varphi \in W_{0}^{1,2}(\Omega),
$$

which is the first half of (2.10). As for the second, by Remark 2.2 we have that $u$ is a weak solution of the first equation of (2.1). Choosing $u-v$ as test function, with $v$ in $W_{0}^{1,2}(\Omega)$ such that $\psi|v|^{r}$ belongs to $L^{1}(\Omega)$, we obtain that

$$
\int_{\Omega}\left[a(x)+|\nabla u|^{2}\right] \nabla u \nabla(u-v)+\int_{\Omega} \psi|u|^{r-2} u(u-v)=\int_{\Omega} f(u-v) .
$$

Adding to both sides of this identity the term

$$
\frac{1}{2} \int_{\Omega} a(x)|\nabla v|^{2},
$$

we obtain after straightforward passages that

$$
\begin{aligned}
& \frac{1}{2} \int_{\Omega} a(x)|\nabla(u-v)|^{2}+\frac{1}{2} \int_{\Omega} a(x)|\nabla u|^{2}+\left[\int_{\Omega}|\nabla u|^{2}\right] \int_{\Omega} \nabla u \nabla(u-v) \\
& \quad+\int_{\Omega} \psi|u|^{r-2} u(u-v)-\int_{\Omega} f u=\frac{1}{2} \int_{\Omega} a(x)|\nabla u|^{2}-\int_{\Omega} f u .
\end{aligned}
$$

Dropping a positive term, we therefore obtain that

$$
\begin{aligned}
& \frac{1}{2} \int_{\Omega} a(x)|\nabla u|^{2}+\left[\int_{\Omega}|\nabla u|^{2}\right] \int_{\Omega} \nabla u \nabla(u-v)+\int_{\Omega} \psi|u|^{r-2} u(u-v)-\int_{\Omega} f u \\
& \quad \leq \frac{1}{2} \int_{\Omega} a(x)|\nabla u|^{2}-\int_{\Omega} f v .
\end{aligned}
$$

Observe now that since $r>1$, the function $t \mapsto|t|^{r}$ is convex; therefore,

$$
|s|^{r} \geq|t|^{r}+r|t|^{r-2} t(s-t), \quad \forall s, t \in \mathbb{R} .
$$

Writing this inequality with $s=v(x)$ and $t=u(x)$, we therefore have that

$$
|v(x)|^{r} \geq|u(x)|^{r}+r|u(x)|^{r-2} u(x)(v(x)-u(x)),
$$

which can be rewritten, integrating on $\Omega$ and recalling that $\psi \geq 0$, and that $\psi|v|^{r}$ belongs to $L^{1}(\Omega)$, as

$$
\int_{\Omega} \psi|u|^{r-2} u(u-v) \geq \frac{1}{r} \int_{\Omega} \psi|u|^{r}-\frac{1}{r} \int_{\Omega} \psi|v|^{r} .
$$

Substituting in (2.11), we obtain (since $\psi \geq 0$, we have $\psi=\psi^{+}$)

$$
\begin{aligned}
& \frac{1}{2} \int_{\Omega} a(x)|\nabla u|^{2}+\left[\int_{\Omega}|\nabla u|^{2}\right] \int_{\Omega} \nabla u \nabla(u-v)+\frac{1}{r} \int_{\Omega} \psi^{+}|u|^{r}-\int_{\Omega} f u \\
& \quad \leq \frac{1}{2} \int_{\Omega} a(x)|\nabla u|^{2}+\frac{1}{r} \int_{\Omega} \psi^{+}|v|^{r}-\int_{\Omega} f v .
\end{aligned}
$$

It only remains to deal with the term

$$
\left[\int_{\Omega}|\nabla u|^{2}\right] \int_{\Omega} \nabla u \nabla(u-v) .
$$

We begin by adding and subtracting the quantity

$$
\frac{1}{2}\left[\int_{\Omega}|\nabla u|^{2}\right] \int_{\Omega}|\nabla v|^{2}
$$

to obtain

$$
\frac{1}{2}\left[\int_{\Omega}|\nabla u|^{2}\right] \int_{\Omega}|\nabla(u-v)|^{2}+\frac{1}{2}\left[\int_{\Omega}|\nabla u|^{2}\right]\left[\int_{\Omega}|\nabla u|^{2}-\int_{\Omega}|\nabla v|^{2}\right],
$$


so that

$$
\left[\int_{\Omega}|\nabla u|^{2}\right] \int_{\Omega} \nabla u \nabla(u-v) \geq \frac{1}{2}\left[\int_{\Omega}|\nabla u|^{2}\right]\left[\int_{\Omega}|\nabla u|^{2}-\int_{\Omega}|\nabla v|^{2}\right] .
$$

Since

$$
t(t-s) \geq \frac{1}{2} t^{2}-\frac{1}{2} s^{2} \quad \forall s, t \in \mathbb{R},
$$

writing this inequality with $s=\int_{\Omega}|\nabla v|^{2}$ and $t=\int_{\Omega}|\nabla u|^{2}$, we have that

$$
\left[\int_{\Omega}|\nabla u|^{2}\right]\left[\int_{\Omega}|\nabla u|^{2}-\int_{\Omega}|\nabla v|^{2}\right] \geq \frac{1}{2}\left[\int_{\Omega}|\nabla u|^{2}\right]^{2}-\frac{1}{2}\left[\int_{\Omega}|\nabla v|^{2}\right]^{2} .
$$

Therefore, using (2.13) we deduce that

$$
\begin{aligned}
& {\left[\int_{\Omega}|\nabla u|^{2}\right] \int_{\Omega} \nabla u \nabla(u-v) \geq \frac{1}{2}\left[\int_{\Omega}|\nabla u|^{2}\right]\left[\int_{\Omega}|\nabla u|^{2}-\int_{\Omega}|\nabla v|^{2}\right]} \\
& \quad \geq \frac{1}{4}\left[\int_{\Omega}|\nabla u|^{2}\right]^{2}-\frac{1}{4}\left[\int_{\Omega}|\nabla v|^{2}\right]^{2} .
\end{aligned}
$$

Substituting this inequality in (2.12), we thus have that

$$
\begin{aligned}
& \frac{1}{2} \int_{\Omega} a(x)|\nabla u|^{2}+\frac{1}{4}\left[\int_{\Omega}|\nabla u|^{2}\right]^{2}+\frac{1}{r} \int_{\Omega} \psi^{+}|u|^{r}-\int_{\Omega} f u \\
& \quad \leq \frac{1}{2} \int_{\Omega} a(x)|\nabla u|^{2}+\frac{1}{4}\left[\int_{\Omega}|\nabla v|^{2}\right]^{2}+\frac{1}{r} \int_{\Omega} \psi^{+}|v|^{r}-\int_{\Omega} f v .
\end{aligned}
$$

If we subtract to both sides the term

$$
\frac{1}{2 r} \int_{\Omega} M(x) \nabla \psi \nabla \psi,
$$

which is finite (recall that $\psi$ belongs to $W_{0}^{1,2}(\Omega)$ ), we obtain that

$$
J(u, \psi) \leq J(v, \psi), \quad \forall v \in W_{0}^{1,2}(\Omega) \text { such that } \psi|v|^{r} \in L^{1}(\Omega),
$$

which is the second part of $(2.10)$.

\section{Outside The ENERGy SPACE}

Up to now, we have made assumptions on $f$ which guaranteed the existence of finite energy solutions (i.e., in $\left.W_{0}^{1,2}(\Omega)\right) u$ and $\psi$ to the system. These assumptions were necessary in order to give a meaning to the term $\int_{\Omega}|\nabla u|^{2}$ which appears in the first equation. What happens if these assumptions are violated?

In order to give an idea of what may happen, we will confine ourselves to a particular case: we suppose that $f \geq 0$ belongs to $L^{1}(\Omega) \backslash W^{-1,2}(\Omega)$, and that

$$
1<r \leq \frac{N+2}{N-2}
$$

Our result is the following:

Proposition 3.1. Let $a$ and $M$ be such that (1.4) and (1.5) hold. Let $r>1$ be such that (3.1) holds, and let $f \geq 0$ in $L^{1}(\Omega) \backslash W^{-1,2}(\Omega)$. If $\left\{u_{n}\right\}$ and $\left\{\psi_{n}\right\}$ are sequences of solutions of $(2.2)$, with $f_{n}=T_{n}(f)$, then

$$
\text { the sequence }\left\{u_{n}\right\} \text { is unbounded in } W_{0}^{1,2}(\Omega) \text {. }
$$


Proof. Let $u_{n}$ and $\psi_{n}$ be solutions of (2.2), define

$$
D_{n}=\int_{\Omega}\left|\nabla u_{n}\right|^{2}
$$

and suppose by contradiction that the sequence $\left\{D_{n}\right\}$ is bounded in $\mathbb{R}$. Hence, the sequence $\left\{u_{n}\right\}$ is bounded in $W_{0}^{1,2}(\Omega)$, and let $u$ be the weak limit of (a subsequence of) $u_{n}$ in $W_{0}^{1,2}(\Omega)$. Since the sequence $\left\{u_{n}\right\}$ is bounded in $L^{2^{*}}(\Omega)$ by Sobolev embedding, the sequence $\left\{\left|u_{n}\right|^{r}\right\}$ is bounded in $L^{2^{*} / r}(\Omega)$; by (3.1), we have that

$$
\frac{2^{*}}{r} \geq \frac{2 N}{N-2} \frac{N-2}{N+2}=\frac{2 N}{N+2},
$$

so that, by elliptic estimates, we have that the sequence $\left\{\psi_{n}\right\}$ is bounded in $W_{0}^{1,2}(\Omega)$ and in $L^{2^{*}}(\Omega)$. Therefore, the sequence $\left\{\psi_{n}\left|u_{n}\right|^{r-1}\right\}$ is bounded in $L^{\sigma}(\Omega)$, with

$$
\frac{1}{\sigma}=\frac{1}{2^{*}}+\frac{r-1}{2^{*}}=\frac{r}{2^{*}} \leq \frac{N+2}{N-2} \frac{N-2}{2 N}=\frac{N+2}{2 N},
$$

where in the last passage we have used (3.1). Therefore,

$$
\sigma \geq \frac{2 N}{N+2}
$$

and so the above estimates are enough to pass to the limit in the first equation of (2.2). We obtain that $u$ is a solution of

$$
u \in W_{0}^{1,2}(\Omega):-\operatorname{div}([a(x)+D] \nabla u)+\psi|u|^{r-2} u=f,
$$

where $D$ is the limit of (a subsequence of) $D_{n}$ (note that it is not necessarily true that $D=\int_{\Omega}|\nabla u|^{2}$ since we only have weak convergence). This is, however, not possible: indeed, since $u$ belongs to $W_{0}^{1,2}(\Omega)$ and $a(x)$ is bounded, we have that $-\operatorname{div}([a(x)+$ $D] \nabla u$ ) belongs to $W^{-1,2}(\Omega)$; since $\psi|u|^{r-2} u$ belongs (at least) to $L^{\frac{2 N}{N+2}}(\Omega)$, we have that it is in $W^{-1,2}(\Omega)$ as well; therefore, from the equation we obtain that $f$ belongs to $W^{-1,2}(\Omega)$, contradicting our assumption.

By the previous result, the sequence $\left\{u_{n}\right\}$ of solutions of the first equation of (2.2) is not bounded in $W_{0}^{1,2}(\Omega)$. What can be deduced from this information? To simplify the presentation, suppose from now on that

$$
N=6,
$$

so that (3.1) becomes $1<r<2$. Our result is the following.

Proposition 3.2. Let $a$ and $M$ be such that (1.4) and (1.5) hold. Let $N=6$, let $1<r<2$, and let $f \geq 0$ in $L^{1}(\Omega) \backslash W^{-1,2}(\Omega)$. If $\left\{u_{n}\right\}$ and $\left\{\psi_{n}\right\}$ are sequences of solutions of $(2.2)$, with $f_{n}=T_{n}(f)$, then the sequence $\left\{T_{k}\left(u_{n}\right)\right\}$ is bounded in $W_{0}^{1,2}(\Omega)$ for every $k>0$, and the sequence $\left\{u_{n}\right\}$ is bounded in $W_{0}^{1, q}(\Omega)$, for every $1<q<\frac{18}{11}$. Furthermore, if

$$
D_{n}=\int_{\Omega}\left|\nabla u_{n}\right|^{2}
$$

then the sequence $\left\{T_{k}\left(D_{n} u_{n}\right)\right\}$ is bounded in $W_{0}^{1,2}(\Omega)$, and the sequence $\left\{D_{n} u_{n}\right\}$ is bounded in $W_{0}^{1, \rho}(\Omega)$, for every $1<\rho<\frac{6}{5}$. Finally, the sequence $\left\{u_{n}\right\}$ weakly converges to zero in $W_{0}^{1, q}(\Omega)$, for every $1<q<\frac{18}{11}$, while the sequence $\left\{D_{n} u_{n}\right\}$ weakly converges 
to a function $w$ in $W_{0}^{1, \rho}(\Omega)$, for every $1<\rho<\frac{6}{5}$, with $w$ the entropy solution of the equation

$$
-\Delta w=f .
$$

That is, $w$ is such that $T_{k}(w)$ belongs to $W_{0}^{1,2}(\Omega)$ for every $k>0$, and

$$
\int_{\Omega} \nabla w \nabla T_{k}(w-\varphi) \leq \int_{\Omega} f T_{k}(w-\varphi), \quad \forall k>0, \forall \varphi \in W_{0}^{1,2}(\Omega) \cap L^{\infty}(\Omega) .
$$

As for the second equation of (2.2), it degenerates to the identity $0=0$.

Proof. Let $k>0$, and choose $T_{k}\left(u_{n}\right)$ as test function in the first equation of (2.2). Using (1.4), dropping two positive terms (note here that we keep the part with the integral of $\left|\nabla u_{n}\right|^{2}$ ), and recalling that $0 \leq f_{n} \leq f$ we obtain

$$
\left[\int_{\Omega}\left|\nabla u_{n}\right|^{2}\right] \int_{\Omega}\left|\nabla T_{k}\left(u_{n}\right)\right|^{2} \leq \int_{\Omega} f_{n} T_{k}\left(u_{n}\right) \leq k\|f\|_{L^{1}(\Omega)} .
$$

Since

$$
\int_{\Omega}\left|\nabla u_{n}\right|^{2} \geq \int_{\Omega}\left|\nabla T_{k}\left(u_{n}\right)\right|^{2}
$$

we therefore obtain from (3.4) that

$$
\int_{\Omega}\left|\nabla T_{k}\left(u_{n}\right)\right|^{2} \leq C k^{\frac{1}{2}}
$$

which implies that, for $k$ fixed,

$$
\left\{T_{k}\left(u_{n}\right)\right\} \text { is bounded in } W_{0}^{1,2}(\Omega) .
$$

Using Sobolev inequality, and observing that $T_{k}\left(u_{n}\right)=k$ on the set $\left\{u_{n} \geq k\right\}$, we thus have (here we follow [4])

$\mathcal{S} k^{2} \operatorname{meas}\left(\left\{u_{n} \geq k\right\}\right)^{\frac{2}{2^{*}}} \leq \mathcal{S}\left[\int_{u_{n} \geq k}\left|T_{k}\left(u_{n}\right)\right|^{2^{*}}\right]^{\frac{2}{2^{*}}} \leq \mathcal{S}\left[\int_{\Omega}\left|T_{k}\left(u_{n}\right)\right|^{2^{*}}\right]^{\frac{2}{2^{*}}} \leq \int_{\Omega}\left|\nabla T_{k}\left(u_{n}\right)\right|^{2}$,

which, together with (3.5), implies that

$$
\operatorname{meas}\left(\left\{u_{n} \geq k\right\}\right)^{\frac{2}{2^{*}}} \leq \frac{C}{k^{\frac{3}{2}}},
$$

that is (recall that $N=6$, so that $2^{*}=3$ )

$$
\operatorname{meas}\left(\left\{u_{n} \geq k\right\}\right) \leq \frac{C}{k^{\frac{9}{4}}} .
$$

Let now $\lambda>0$; since

$$
\left\{\left|\nabla u_{n}\right| \geq \lambda\right\}=\left\{\left|\nabla u_{n}\right| \geq \lambda, u_{n}<k\right\} \cup\left\{\left|\nabla u_{n}\right| \geq \lambda, u_{n} \geq k\right\},
$$

we have that

$$
\begin{aligned}
\operatorname{meas}\left(\left\{\left|\nabla u_{n}\right| \geq \lambda\right\}\right) & \leq \operatorname{meas}\left(\left\{\left|\nabla u_{n}\right| \geq \lambda, u_{n}<k\right\}\right)+\operatorname{meas}\left(\left\{\left|\nabla u_{n}\right| \geq \lambda, u_{n} \geq k\right\}\right) \\
& \leq \frac{1}{\lambda^{2}} \int_{\Omega}\left|\nabla T_{k}\left(u_{n}\right)\right|^{2}+\operatorname{meas}\left(\left\{u_{n} \geq k\right\}\right) \leq \frac{C k^{\frac{1}{2}}}{\lambda^{2}}+\frac{C}{k^{\frac{9}{4}}} .
\end{aligned}
$$

Choosing $k^{\frac{1}{2}}=\lambda^{\frac{4}{11}}$ (so that the two terms in the right hand side have the same behaviour), we obtain

$$
\operatorname{meas}\left(\left\{\left|\nabla u_{n}\right| \geq \lambda\right\}\right) \leq \frac{C}{\lambda^{\frac{18}{11}}}
$$


Estimates (3.7) and (3.8) imply (thanks to the embeddings between Marcinkiewicz and Lebesgue spaces) that

$$
\left\{u_{n}\right\} \text { is bounded in } W_{0}^{1, q}(\Omega), \forall q<\frac{18}{11} \text {, and in } L^{s}(\Omega), \forall s<\frac{9}{4} .
$$

The boundedness of $\left\{u_{n}\right\}$ in $L^{s}(\Omega)$, implies that $\left\{\left|u_{n}\right|^{r}\right\}$ is bounded in $L^{s / r}(\Omega)$, for every $s<\frac{9}{4}$. Thus, by the elliptic estimates of [11] or [7], applied to the second equation of $(2.2)$, we have that the sequence $\left\{\psi_{n}\right\}$ is bounded in $L^{\rho}(\Omega)$, for every $\rho<\left(\frac{s}{r}\right)^{* *}=\frac{3 s}{3 r-s}$. Therefore, the sequence $\left\{\psi_{n} u_{n}^{r-1}\right\}$ is bounded in $L^{\sigma}(\Omega)$, for every $\sigma$ such that

$$
\frac{1}{\sigma}=\frac{1}{\rho}+\frac{r-1}{s}>\frac{3 r-s}{3 s}+\frac{3 r-3}{3 s}=\frac{6 r-s-3}{3 s} \quad \Longleftrightarrow \quad \sigma<\frac{3 s}{6 r-s-3} .
$$

Note that one has $\sigma>1$ if and only if one can choose $s$ such that $\frac{3 s}{6 r-s-3}>1$, that is if $s>\frac{3(2 r-1)}{4}$. Since by (3.1) we have $r<2$, then $\frac{3(2 r-1)}{4}<\frac{9}{4}$, so that such a choice of $s$ is possible by (3.9).

Summing up, for the first equation one has (3.9) and

$$
\left\{\psi_{n} u_{n}^{r-1}\right\} \text { is bounded in } L^{\sigma}(\Omega) \text {, for some } \sigma>1 \text {. }
$$

Choose now $T_{k}\left(D_{n} u_{n}\right)$ as test function in the first equation, with $D_{n}$ given by (3.3). Dropping two positive terms (as before), and recalling that $0 \leq f_{n} \leq f$, we obtain

$$
\int_{\Omega}\left|\nabla T_{k}\left(D_{n} u_{n}\right)\right|^{2}=D_{n} \int_{\Omega} \nabla u_{n} \nabla T_{k}\left(D_{n} u_{n}\right) \leq \int_{\Omega} f_{n} T_{k}\left(D_{n} u_{n}\right) \leq k\|f\|_{L^{1}(\Omega)} .
$$

This inequality implies that for $k$ fixed the sequence $\left\{T_{k}\left(D_{n} u_{n}\right)\right\}$ is bounded in $W_{0}^{1,2}(\Omega)$, and, reasoning as in [4] (or as above), that the sequence $\left\{D_{n} u_{n}\right\}$ is bounded in $W_{0}^{1, \rho}(\Omega)$, for every $1<\rho<\frac{6}{5}$. Note that $\frac{18}{11}>\frac{6}{5}$, so that the estimate (3.9) on $u_{n}$ is "better" than that on $D_{n} u_{n}$.

Since the sequence $\left\{D_{n}\right\}$ is not bounded by Proposition 3.1, and the sequence $\left\{D_{n} u_{n}\right\}$ is bounded in $W_{0}^{1, \rho}(\Omega), 1<\rho<\frac{6}{5}$, it follows that (up to subsequences) $u_{n}$ tends to zero in the same space (since the limit is independent on the subsequence, then the whole sequence $\left\{u_{n}\right\}$ tends to zero); therefore, $u_{n}$ tends to zero in the smaller space $W_{0}^{1, q}(\Omega)$, $1<q<\frac{18}{11}$; furthermore, the sequence $\left\{\left|u_{n}\right|^{r}\right\}$, and so by elliptic estimates the sequence $\left\{\psi_{n}\right\}$, tend to zero. This fact means that the second equation becomes the identity $0=0$ (i.e., there is no limit equation). As for the first one, the term $\psi_{n}\left|u_{n}\right|^{r-1}$ tends to zero, while the estimates on $T_{k}\left(u_{n}\right)$ (see (3.6)), on $D_{n} u_{n}$ and on $T_{k}\left(D_{n} u_{n}\right)$ yield that $T_{k}\left(u_{n}\right)$ tends to zero weakly in $W_{0}^{1,2}(\Omega)$, that $D_{n} u_{n}$ converges to some function $w$ weakly in $W_{0}^{1, q}(\Omega), 1<q<\frac{6}{5}$, and that $T_{k}\left(D_{n} u_{n}\right)$ converges to $T_{k}(w)$ weakly in $W_{0}^{1,2}(\Omega)$.

We now follow again the approach of [4], and choose $T_{k}\left(D_{n} u_{n}-\varphi\right)$ as test function in the first equation of $(2.2)$, with $\varphi$ in $W_{0}^{1,2}(\Omega) \cap L^{\infty}(\Omega)$. We obtain

$$
\begin{gathered}
\int_{\Omega} a(x) \nabla u_{n} \nabla T_{k}\left(D_{n} u_{n}-\varphi\right)+D_{n} \int_{\Omega} \nabla u_{n} \nabla T_{k}\left(D_{n} u_{n}-\varphi\right) \\
\quad+\int_{\Omega} \psi_{n}\left|u_{n}\right|^{r-2} u_{n} T_{k}\left(D_{n} u_{n}-\varphi\right)=\int_{\Omega} f_{n} T_{k}\left(D_{n} u_{n}-\varphi\right) .
\end{gathered}
$$

The convergences proved so far yield that

$$
\lim _{n \rightarrow+\infty} \int_{\Omega} \psi_{n}\left|u_{n}\right|^{r-2} u_{n} T_{k}\left(D_{n} u_{n}-\varphi\right)=0, \quad \lim _{n \rightarrow+\infty} \int_{\Omega} f_{n} T_{k}\left(D_{n} u_{n}-\varphi\right)=\int_{\Omega} f T_{k}(w-\varphi) .
$$


As for the first term, we have

$$
\begin{gathered}
\int_{\Omega} a(x) \nabla u_{n} \nabla T_{k}\left(D_{n} u_{n}-\varphi\right)=\frac{1}{D_{n}} \int_{\Omega} a(x) \nabla\left(D_{n} u_{n}\right) \nabla T_{k}\left(D_{n} u_{n}-\varphi\right) \\
=\frac{1}{D_{n}} \int_{\Omega} a(x)\left|\nabla T_{k}\left(D_{n} u_{n}-\varphi\right)\right|^{2}+\frac{1}{D_{n}} \int_{\Omega} a(x) \nabla \varphi \nabla T_{k}\left(D_{n} u_{n}-\varphi\right) .
\end{gathered}
$$

Since $D_{n}$ diverges, and the two integrals are bounded with respect to $n$, we have that

$$
\lim _{n \rightarrow+\infty} \int_{\Omega} a(x) \nabla u_{n} \nabla T_{k}\left(D_{n} u_{n}-\varphi\right)=0 .
$$

As for the second integral, we have

$$
\begin{aligned}
\int_{\Omega} & \nabla\left(D_{n} u_{n}\right) \nabla T_{k}\left(D_{n} u_{n}-\varphi\right)=\int_{\Omega}\left|\nabla T_{k}\left(D_{n} u_{n}-\varphi\right)\right|^{2} \\
& +\int_{\Omega} \nabla \varphi \nabla T_{k}\left(D_{n} u_{n}-\varphi\right) \geq \int_{\Omega} \nabla \varphi \nabla T_{k}\left(D_{n} u_{n}-\varphi\right),
\end{aligned}
$$

so that

$$
\liminf _{n \rightarrow+\infty} \int_{\Omega} \nabla\left(D_{n} u_{n}\right) \nabla T_{k}\left(D_{n} u_{n}-\varphi\right) \geq \int_{\Omega} \nabla \varphi \nabla T_{k}(w-\varphi) .
$$

Putting together all the convergence results, we thus have that

$$
\int_{\Omega} \nabla \varphi \nabla T_{k}(w-\varphi) \leq \int_{\Omega} f T_{k}(w-\varphi), \quad \forall k>0, \forall \varphi \in W_{0}^{1,2}(\Omega) \cap L^{\infty}(\Omega) .
$$

Thanks to the results of [8], we therefore have that

$$
\int_{\Omega} \nabla w \nabla T_{k}(w-\varphi)=\int_{\Omega} f T_{k}(w-\varphi), \quad \forall k>0, \forall \varphi \in W_{0}^{1,2}(\Omega) \cap L^{\infty}(\Omega),
$$

that is, $w$ is the entropy solution (see [4]) of the equation

$$
-\Delta w=f,
$$

as desired.

\section{APPENDIX: BASIC RESULTS AND EXISTENCE FOR BOUNDED DATA}

In this Appendix, we will prove some results concerning the first equation of system (1.3), and the whole system in the case of bounded data.

Proposition 3.3. Let $v$ be a function in $W_{0}^{1,2}(\Omega)$, let $\varphi \geq 0$ be a function in $L^{1}(\Omega)$, let $f$ be a function in $L^{\infty}(\Omega)$, and let $r>1$. Then there exists a unique weak solution $u$ of

$$
u \in W_{0}^{1,2}(\Omega):-\operatorname{div}\left(\left[a(x)+\int_{\Omega}|\nabla v|^{2}\right] \nabla u\right)+\varphi|u|^{r-2} u=f,
$$

that is, $\varphi|u|^{r}$ belongs to $L^{1}(\Omega)$, and

$$
\int_{\Omega}\left[a(x)+\int_{\Omega}|\nabla v|^{2}\right] \nabla u \nabla \eta+\int_{\Omega} \varphi|u|^{r-2} u \eta=\int_{\Omega} f \eta, \quad \forall \eta \in W_{0}^{1,2}(\Omega) \cap L^{\infty}(\Omega) .
$$

Furthermore,

$$
\|u\|_{W_{0}^{1,2}(\Omega)}+\|u\|_{L^{\infty}(\Omega)} \leq C\|f\|_{L^{1}(\Omega) 73 \infty},
$$

for some positive constant $C$ independent on $v$. 
Proof. The existence and uniqueness of a solution $u$ in $W_{0}^{1,2}(\Omega)$ of (3.11) follows from well-known results on semilinear elliptic equations (see, for example, [5]) since (3.11) is of the kind

$$
-\operatorname{div}(Q(x) \nabla w)+b(x)|w|^{r-2} w=g,
$$

with $Q$ a uniformly elliptic and bounded matrix, $r>1, b$ a function in $L^{1}(\Omega)$, and $g$ a function belonging to some Lebesgue space.

As for (3.12), we begin by choosing $T_{k}(u)$ as test function. Dropping two positive terms, and using (1.4), we obtain

$$
\alpha \int_{\Omega}\left|\nabla T_{k}(u)\right|^{2} \leq \int_{\Omega} f T_{k}(u) .
$$

Letting $k$ tend to infinity, an using Fatou lemma in the left hand side, and Lebesgue theorem in the right hand one, we obtain

$$
\alpha \int_{\Omega}|\nabla u|^{2} \leq \int_{\Omega} f u
$$

and from this inequality it is easy to prove (using Sobolev embedding and Hölder inequality) that

$$
\|u\|_{W_{0}^{1,2}(\Omega)} \leq C\|f\|_{L^{\infty}(\Omega)},
$$

which is one half of (3.12). The second half can be obtained by choosing $G_{k}\left(T_{h}(u)\right)$, letting $h$ tend to infinity, and then following the proof of Théorème 4.1 of the paper [11] by G. Stampacchia.

We can now prove an existence result for solutions of (1.3), in the case of bounded data $f$.

TheOREm 3.4. Let $f$ be a function in $L^{\infty}(\Omega)$, and let $r>1$. Then there exist $u$ and $\psi$, weak solutions of the system

$$
\left\{\begin{array}{l}
u \in W_{0}^{1,2}(\Omega):-\operatorname{div}\left(\left[a(x)+\int_{\Omega}|\nabla u|^{2}\right] \nabla u\right)+\psi|u|^{r-2} u=f, \\
\psi \in W_{0}^{1,2}(\Omega):-\operatorname{div}(M(x) \nabla \psi)=|u|^{r} .
\end{array}\right.
$$

Furthermore, $u$ and $\psi$ belong to $L^{\infty}(\Omega)$, and $\psi \geq 0$.

Proof. Let $\sigma$ in $\mathbb{N}$ and $v$ in $W_{0}^{1,2}(\Omega)$; by Lax-Milgram theorem, there exists a unique solution $\varphi$ of

$$
\varphi \in W_{0}^{1,2}(\Omega):-\operatorname{div}(M(x) \nabla \varphi)=\left|T_{\sigma}(v)\right|^{r} .
$$

By standard elliptic estimates, one has that

$$
\|\varphi\|_{W_{0}^{1,2}(\Omega)} \leq C \sigma^{r}
$$

for some positive constant $C$. By the maximum principle, $\varphi \geq 0$, so that, by Proposition 3.3 , there exists a unique solution $u$ of

$$
u \in W_{0}^{1,2}(\Omega):-\operatorname{div}\left(\left[a(x)+\int_{\Omega}|\nabla v|^{2}\right] \nabla u\right)+\varphi|u|^{r-2} u=f .
$$

Since, by (3.12), one has

$$
\|u\|_{W_{0}^{1,2}(\Omega)} \leq C\|f\|_{L^{\infty}(\Omega)} \stackrel{\text { def }}{=} R,
$$


the ball $B_{R}(0)$ of $W_{0}^{1,2}(\Omega)$ is invariant for the map $S: v \mapsto u$. We are going to prove that the map $S$ is completely continuous

We begin by proving that if $\left\{v_{n}\right\}$ is bounded in $W_{0}^{1,2}(\Omega)$, then there exists a subsequence of $\left\{u_{n}=S\left(v_{n}\right)\right\}$ which is strongly convergent in $W_{0}^{1,2}(\Omega)$. Indeed, since the ball $B_{R}(0)$ is invariant for $S$, the sequence $\left\{u_{n}\right\}$ is bounded in $W_{0}^{1,2}(\Omega)$; hence, up to subsequences it will converge, weakly in $W_{0}^{1,2}(\Omega)$, strongly in (for example) $L^{2}(\Omega)$, and almost everywhere in $\Omega$, to a function $u$. Since by (3.12) the sequence $\left\{u_{n}\right\}$ is bounded in $L^{\infty}(\Omega)$, we have that $u_{n}$ strongly converges to $u$ in $L^{p}(\Omega)$ for every $p \geq 1$. Furthermore, if $\varphi_{n}$ is the solution of (3.14) with datum $\left|T_{\sigma}\left(v_{n}\right)\right|^{r}$, then by (3.15) the sequence $\left\{\varphi_{n}\right\}$ is bounded in $W_{0}^{1,2}(\Omega)$, so that, up to subsequences, it strongly converges to some function $\varphi$ in (for example) $L^{2}(\Omega)$.

We now choose $u_{n}-u$ as test function in the equation solved by $u_{n}$ to have that

$$
\int_{\Omega}\left[a(x)+\int_{\Omega}\left|\nabla v_{n}\right|^{2}\right] \nabla u_{n} \nabla\left(u_{n}-u\right)+\int_{\Omega} \varphi_{n}\left|u_{n}\right|^{r-2} u_{n}\left(u_{n}-u\right)=\int_{\Omega} f\left(u_{n}-u\right) .
$$

Adding and subtracting the term

$$
\int_{\Omega}\left[a(x)+\int_{\Omega}\left|\nabla v_{n}\right|^{2}\right] \nabla u \nabla\left(u_{n}-u\right)
$$

we obtain, using (1.4) and dropping a positive term,

$$
\begin{aligned}
& \alpha \int_{\Omega}\left|\nabla\left(u_{n}-u\right)\right|^{2}+\int_{\Omega} \varphi_{n}\left|u_{n}\right|^{r-2} u_{n}\left(u_{n}-u\right) \\
& \quad \leq \int_{\Omega} f\left(u_{n}-u\right)+\int_{\Omega}\left[a(x)+\int_{\Omega}\left|\nabla v_{n}\right|^{2}\right] \nabla u \nabla\left(u_{n}-u\right) .
\end{aligned}
$$

Since $f$ belongs to $L^{\infty}(\Omega)$, and $u_{n}-u$ tends to zero in (for example) $L^{1}(\Omega)$, we have that

$$
\lim _{n \rightarrow+\infty} \int_{\Omega} f\left(u_{n}-u\right)=0
$$

while, since $a(x) \nabla u$ belongs to $\left(L^{2}(\Omega)\right)^{N},\left\{v_{n}\right\}$ is bounded in $W_{0}^{1,2}(\Omega)$, and $\nabla\left(u_{n}-u\right)$ tends to zero weakly in $\left(L^{2}(\Omega)\right)^{N}$, we have that

$$
\begin{aligned}
\lim _{n \rightarrow+\infty} \int_{\Omega}\left[a(x)+\int_{\Omega}\left|\nabla v_{n}\right|^{2}\right] \nabla u \nabla\left(u_{n}-u\right) & =\lim _{n \rightarrow+\infty} \int_{\Omega} a(x) \nabla u \nabla\left(u_{n}-u\right) \\
+ & \lim _{n \rightarrow+\infty}\left[\int_{\Omega}\left|\nabla v_{n}\right|^{2}\right] \int_{\Omega} \nabla u \nabla\left(u_{n}-u\right)=0 .
\end{aligned}
$$

On the other hand, since $\varphi_{n}$ is strongly convergent to $\varphi$ in $L^{2}(\Omega)$, and $\left|u_{n}\right|^{r-2} u_{n}\left(u_{n}-u\right)$ is strongly convergent to zero in $L^{2}(\Omega)$ as well (recall that $\left\{u_{n}\right\}$ strongly converges to $u$ in every $\left.L^{p}(\Omega)\right)$, we have that

$$
\lim _{n \rightarrow+\infty} \int_{\Omega} \varphi_{n}\left|u_{n}\right|^{r-2} u_{n}\left(u_{n}-u\right)=0 .
$$

These three convergences imply that

$$
\lim _{n \rightarrow+\infty} \alpha \int_{\Omega}\left|\nabla\left(u_{n}-u\right)\right|^{2}=0
$$

so that the sequence $\left\{u_{n}=S\left(v_{n}\right)\right\}$ is strongly convergent in $W_{0}^{1,2}(\Omega)$, up to subsequences. This proves that the map $S$ is compact. To prove its continuity, let $\left\{v_{n}\right\}$ be a 
sequence strongly convergent to $v$ in $W_{0}^{1,2}(\Omega)$, and let $u_{n}=S\left(v_{n}\right)$. If $\varphi_{n}$ is the solution of

$$
\varphi_{n} \in W_{0}^{1,2}(\Omega):-\operatorname{div}\left(M(x) \nabla \varphi_{n}\right)=\left|T_{\sigma}\left(v_{n}\right)\right|^{r},
$$

since $\left\{\left|T_{\sigma}\left(v_{n}\right)\right|^{r}\right\}$ is strongly convergent in (for example) $L^{2}(\Omega)$, then $\varphi_{n}$ is strongly convergent in $W_{0}^{1,2}(\Omega)$ to $\varphi$, the unique solution of

$$
\varphi \in W_{0}^{1,2}(\Omega):-\operatorname{div}(M(x) \nabla \varphi)=\left|T_{\sigma}(v)\right|^{r} .
$$

On the other hand, since the sequence $\left\{v_{n}\right\}$ is bounded in $W_{0}^{1,2}(\Omega)$, the result proved before yields that, up to subsequences, the sequence $\left\{u_{n}\right\}$ is strongly convergent in $W_{0}^{1,2}(\Omega)$ to some function $u$, and the sequence $\left\{\varphi_{n}\left|u_{n}\right|^{r-2} u_{n}\right\}$ is strongly convergent in (at least) $L^{1}(\Omega)$ to $\varphi|u|^{r-2} u$. Therefore, if $\eta$ belongs to $W_{0}^{1,2}(\Omega) \cap L^{\infty}(\Omega)$, one can pass to the limit in $n$ in the identities

$$
\int_{\Omega}\left[a(x)+\int_{\Omega}\left|\nabla v_{n}\right|^{2}\right] \nabla u_{n} \nabla \eta+\int_{\Omega} \varphi_{n}\left|u_{n}\right|^{r-2} u_{n} \eta=\int_{\Omega} f \eta,
$$

to have that $u$ is such that

$$
\int_{\Omega}\left[a(x)+\int_{\Omega}|\nabla v|^{2}\right] \nabla u \nabla \eta+\int_{\Omega} \varphi|u|^{r-2} u \eta=\int_{\Omega} f \eta, \quad \forall \eta \in W_{0}^{1,2}(\Omega) \cap L^{\infty}(\Omega) .
$$

This implies, by uniqueness of the solution, that $u=S(v)$, and that the whole sequence $\left\{u_{n}\right\}$ is strongly convergent in $W_{0}^{1,2}(\Omega)$ to $u$; therefore, $S$ is continuous, and so $S$ is completely continuous.

Therefore, by Schauder fixed point theorem there exists $u$ in $W_{0}^{1,2}(\Omega)$ such that $S(u)=u$; if we define $\psi$ as the unique solution of

$$
\psi \in W_{0}^{1,2}(\Omega):-\operatorname{div}(M(x) \nabla \psi)=\left|T_{\sigma}(u)\right|^{r},
$$

we have proved that for every $\sigma$ in $\mathbb{N}$ there exist weak solutions $u_{\sigma}$ and $\psi_{\sigma}$ of the system

$$
\left\{\begin{array}{l}
u_{\sigma} \in W_{0}^{1,2}(\Omega):-\operatorname{div}\left(\left[a(x)+\int_{\Omega}\left|\nabla u_{\sigma}\right|^{2}\right] \nabla u_{m}\right)+\psi_{\sigma}\left|u_{\sigma}\right|^{r-2} u_{\sigma}=f, \\
\psi_{\sigma} \in W_{0}^{1,2}(\Omega):-\operatorname{div}\left(M(x) \nabla \psi_{\sigma}\right)=\left|T_{\sigma}\left(u_{\sigma}\right)\right|^{r}
\end{array}\right.
$$

We observe now that, by (3.12), we have that

$$
\left\|u_{\sigma}\right\|_{L^{\infty}(\Omega)} \leq C\|f\|_{L^{\infty}(\Omega)} \stackrel{\text { def }}{=} R \text {. }
$$

Therefore, if $\sigma \geq R$ we have $T_{\sigma}\left(u_{\sigma}\right)=u_{\sigma}$, so that the functions $u \stackrel{\text { def }}{=} u_{\sigma}$ and $\psi \stackrel{\text { def }}{=} \psi_{\sigma}$ are weak solutions of the system

$$
\left\{\begin{array}{l}
u \in W_{0}^{1,2}(\Omega):-\operatorname{div}\left(\left[a(x)+\int_{\Omega}|\nabla u|^{2}\right] \nabla u\right)+\psi|u|^{r-2} u=f, \\
\psi \in W_{0}^{1,2}(\Omega):-\operatorname{div}(M(x) \nabla \psi)=|u|^{r} .
\end{array}\right.
$$

Furthermore, both $u$ and $\psi$ (by Théorème 4.1 of [11]) belong to $L^{\infty}(\Omega)$, and $\psi \geq 0$, as desired.

\section{ACKNOWLDEGMENTS}

We would like to thank the anonymous referee for the useful comments on the paper. 


\section{REFERENCES}

[1] A. Ambrosetti, D. Arcoya. Remarks on non homogeneous elliptic Kirchhoff equations. NoDEA Nonlinear Differential Equations Appl., 23 (2016), Art. 57, 11.

[2] A. Ambrosetti, D. Arcoya. Positive solutions of elliptic Kirchhoff equations. Adv. Nonlinear Stud., 17 (2017), 3-15.

[3] V. Benci, D. Fortunato. An eigenvalue problem for the Schrödinger-Maxwell equations. Topol. Methods Nonlinear Anal., 11 (1998), 283-293.

[4] P. Bénilan, L. Boccardo, T. Gallouët, R. Gariepy, M. Pierre, J. L. Vázquez. An $L^{1}$-theory of existence and uniqueness of solutions of nonlinear elliptic equations. Ann. Scuola Norm. Sup. Pisa Cl. Sci. (4), 22 (1995), 241-273.

[5] P. Bénilan, H. Brezis. Nonlinear problems related to the Thomas-Fermi equation. J. Evol. Equ., 3 (2003), 673-770. Dedicated to Philippe Bénilan.

[6] L. Boccardo. Elliptic systems of Schrödinger type in the spirit of Benci-Fortunato. Adv. Nonlinear Stud., 15 (2015), 321-331.

[7] L. Boccardo, T. Gallouët. Nonlinear elliptic equations with right-hand side measures. Comm. Partial Differential Equations, 17 (1992), 641-655.

[8] L. Boccardo, L. Orsina. Existence results for Dirichlet problems in $L^{1}$ via Minty's lemma. Appl. Anal., 76 (2000), 309-317.

[9] L. Boccardo, L. Orsina. Regularizing effect for a system of Schrödinger-Maxwell equations. Adv. Calc. Var., 11 (2018), 75-87.

[10] R. Durastanti. Regularizing effect for some p-Laplacian systems. Nonlinear Anal., 188 (2019), 425-438.

[11] G. Stampacchia. Le problème de Dirichlet pour les équations elliptiques du second ordre à coefficients discontinus. Ann. Inst. Fourier (Grenoble), 15 (1965), 189-258.

[12] Q. Zhang. Existence of positive solution to Kirchhoff-Schrödinger-Poisson system with strong singular term. Journal of Mathematical Physics, 60 (2019), 041504.

"SAPIENZA" UnIVERSitÀ Di Roma, ItAly.

Email address: boccardo@mat.uniroma1.it, orsina@mat.uniroma1.it 